\title{
BMJ Open Pharmacist-led intervention to improve medication use in older inpatients using the Drug Burden Index: a study protocol for a before/after intervention with a retrospective control group and multiple case analysis
}

\author{
Marci Elizabeth Dearing (D) ,1,2 Susan Bowles (D) ,2,3 Jennifer Isenor (D) ,3,4 \\ Olga Kits, ${ }^{5,6}$ Lisa Kouladjian O'Donnell (D) , , ${ }^{7,8}$ Heather Neville, ${ }^{2}$ Sarah Hilmer (D) ,7,8 \\ Kent Toombs, ${ }^{2}$ Caroline Sirois (D) , ${ }^{9,10}$ Mohammad Hajizadeh (D) , ${ }^{11}$ Aprill Negus, ${ }^{12}$ \\ Kenneth Rockwood (D) , ${ }^{1,13}$ Emily Reeve (D) ${ }^{1,3,14}$
}

To cite: Dearing ME, Bowles S, Isenor J, et al. Pharmacist-led intervention to improve medication use in older inpatients using the Drug Burden Index: a study protocol for a before/after intervention with a retrospective control group and multiple case analysis. BMJ Open 2020;10:e035656. doi:10.1136/ bmjopen-2019-035656

- Prepublication history for this paper is available online To view these files, please visit the journal online (http://dx.doi org/10.1136/bmjopen-2019035656).

Received 10 November 2019 Revised 17 January 2020 Accepted 28 January 2020

Check for updates

(C) Author(s) (or their employer(s)) 2020. Re-use permitted under CC BY-NC. No commercial re-use. See rights and permissions. Published by BMJ.

For numbered affiliations see end of article.

Correspondence to

Dr Emily Reeve;

Emily.Reeve@unisa.edu.au

\section{ABSTRACT}

Introduction Polypharmacy and potentially inappropriate medication use is common in older adults and is associated with adverse outcomes such as falls and hospitalisations.

Methods and analysis This study is a pharmacist-led medication optimisation initiative using an electronic tool (the Drug Burden Index (DBI) Calculator) in four hospital sites in the Canadian province of Nova Scotia. The study aims to enrol 160 participants between the preintervention and intervention groups. The Standard Protocol Items: Recommendations for Interventional Trials (SPIRIT 2013 checklist) was used to develop the protocol for this prospective interventional implementation study. A preintervention retrospective control cohort and a multiple case study analysis will also be used to assess the effect of intervention implementation. Statistical analysis will involve change in DBI scores and assessment of clinical outcomes, such as rehospitalisation and mortality using appropriate statistical tests including t-test, $\chi^{2}$, analysis of variance and unadjusted and adjusted regression methods. Ethics and dissemination Ethics approval has been granted by the Nova Scotia Health Authority Research Ethics Board. The findings of this study will be published in peer-reviewed journals and presented at local, national and international conferences.

Trial registration number NCT03698487.

\section{INTRODUCTION}

Concerns about adverse effects of medications is a top health priority among older Canadians. ${ }^{12}$ Polypharmacy and inappropriate medication use in older adults is common and associated with a number of harms including adverse drug reactions, falls, hospitalisations, reduced quality of life and mortality. ${ }^{3-6}$ There is particular concern about medications with
Strengths and limitations of this study

The pragmatic design of this intervention, combined with integration of the intervention into workflow, may enhance feasibility, sustainability and generalisability.

- The built-in mixed methods study (multiple case analysis) will lead to new knowledge about implementation, which may be transferrable to other inhospital interventions.

- Due to the pragmatic nature of the study, participants, researchers and those conducting the intervention will not be blinded to the intervention.

- Generalisability to other settings such as community and long-term care will require further exploration.

anticholinergic and sedative effects; older adults may be sensitive to adverse effects of these agents due to changes in pharmacokinetics (increased exposure to the drug) and pharmacodynamics (increased sensitivity to the effects) ${ }^{7-9}$

Anticholinergic medications are used for a variety of conditions such as allergic rhinitis, urinary incontinence and nausea/vomiting. ${ }^{10}$ Other medications, including some common antidepressants, may also have anticholinergic activity even when not central to their efficacy. Sedatives may be used short term to treat insomnia and anxiety; however, many medications produce sedation as an unintended side effect. ${ }^{811}$ While these medications can have therapeutic effects, their use in older adults has been linked to multiple adverse effects, manifesting as limitations in physical and cognitive function. ${ }^{8}$ Furthermore, there 
may be reduced or limited benefits of these agents. ${ }^{78}$ Taken together, in older adults with multimorbidity and polypharmacy, there is a picture of potentially reduced benefit and increased risk associated with use of anticholinergic and sedative medications.

The effects of these medications may be amplified in frail individuals. Frailty is a condition of cumulative reduction in function of multiple body systems. ${ }^{12}$ Frail individuals are vulnerable to external stressors and less able to recover; as such, they are at greater risk of medication related harms. ${ }^{13}$ Despite the knowledge of the risks associated with anticholinergic and sedative medications, their use is relatively common with studies showing use of one or more of these agents in approximately $20 \%-80 \%$ of older adults. ${ }^{8}$ Concerningly, their use may also be more common in frail older adults who are at further risk of harm from medication use. ${ }^{1314}$

The Drug Burden Index (DBI) is an evidence-based pharmacological risk assessment tool developed to measure exposure to anticholinergic and sedative medications, which impair physical and cognitive function. ${ }^{15}$ An increasing DBI score has been associated in several cross-sectional studies internationally with poorer physical function, reduced quality of life, frailty, falls and hospital readmissions in older adults. ${ }^{8}$ Cognitive decline and mortality have been found to be associated with an increased DBI score in some cross-sectional studies but not others. ${ }^{816}$ Longitudinal studies have found that an increased DBI score is independently associated with lower physical function over 5 years, poorer delayed memory performance, increased incidence of frailty and hip fracture, physician visits and mortality. ${ }^{14} 17$ These results represent 20 different studies, span multiple countries (Australia, Canada, Finland, the Netherlands, New Zealand, UK and USA) and provide a substantial argument to reduce DBI score in individuals where possible. ${ }^{8}$

Optimising medication use and reducing exposure to potentially harmful medications through deprescribing (supervised withdrawal of inappropriate medications) may improve outcomes in older adults. Even so, there are numerous barriers to deprescribing such as lack of recognition of potentially harmful medications and limited time of clinicians. ${ }^{18}$ Hospitalisation provides a unique opportunity to initiate deprescribing as there is access to an interdisciplinary team, which can conduct short-term monitoring in a controlled environment. ${ }^{19}$ Pharmacists, in their role as medication experts on the interdisciplinary hospital team, are well positioned to assess medication regimens of hospitalised older adults. ${ }^{19}$ As such they can lead medication optimisation strategies, including deprescribing. ${ }^{20}$

Over 40 different tools have been developed to assess the appropriateness of prescribing and therefore could have potential use in interventions to optimise medication use. ${ }^{21}$ Given the known association of DBI score and patient harm, the DBI has been proposed as an innovative tool to identify older adults at high risk of medication associated harm and to highlight medications that may be suitable for deprescribing. ${ }^{15}$ The DBI was chosen for this study due to the extensive external validation against clinical outcomes. ${ }^{21}$ It is also the only tool that takes into account the specific dose taken by the patient. ${ }^{21}$ Additionally, rather than just highlighting specific medications that are high risk in older adults, it considers the cumulative risk of multiple medications and their doses and provides a score such that the aim can be to reduce the score, rather than stopping specific medications. ${ }^{22}$

The DBI Calculator was developed and validated to automatically calculate the DBI score and produce a detailed report with suggestions to improve the medication regimen of a patient. ${ }^{22}$ In a previous study, $80 \%$ of pharmacists found use of the DBI Calculator an accurate and feasible addition to their practice. ${ }^{22}$ As many older patients in hospital are prescribed large quantities of medications, tools such as the DBI Calculator, may assist pharmacists in targeting deprescribing efforts. ${ }^{23} 24$

The aims of this study are to: (1) determine the effect of integration of an electronic clinical decision support tool (the DBI Calculator) into pharmacist medication optimisation activities on medication changes and clinical outcomes, (2) assess whether there is a variable effect of the intervention based on participant characteristics (ie, frailty status and sex) and/or the setting of the intervention (ie, different ward and/or hospital characteristics) and (3) explore implementation of the DBI Calculator into pharmacist-led medication optimisation activities during inpatient admissions.

\section{METHODS}

\section{Study design}

This project is an in-hospital prospective interventional implementation study across four sites and hospital settings in Nova Scotia, Canada. The study consists of a preintervention control cohort that involves a retrospective chart review of patients discharged from the same ward as the intervention over 1 week prior to intervention start and will be used for comparison with the intervention group. A before/after intervention method was chosen as a pragmatic method to both determine the outcomes of the intervention and explore implementation. Additionally, this method was chosen to minimise contamination bias. A multiple case study (a substudy of the before/after intervention) will also be used to explore the process of implementation and identify factors that shaped implementation.

\section{Study population and recruitment}

Participants will be identified and included as per inclusion and exclusion criteria below if they are admitted to one of the target wards (including both planned and unplanned admissions) during the relevant period for the intervention and control groups. Participants who die during admission will be removed from the study. Those who are transferred to another ward will be considered 


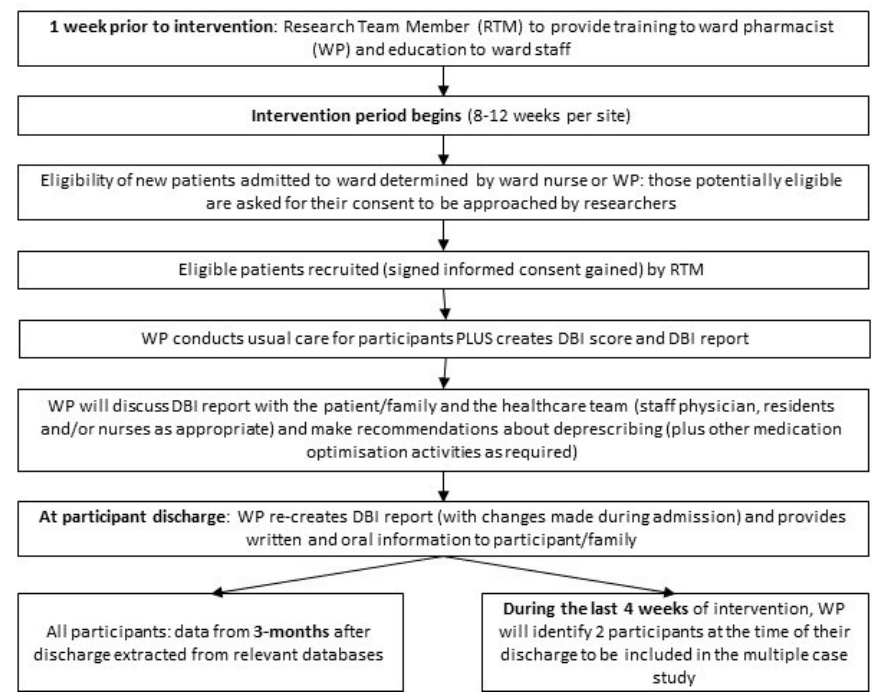

Figure 1 Intervention flow chart.

'discharged' from the ward (but included in the intention to treat analysis).

At each ward, the intervention period will last for 2 months, with training on use of the DBI Calculator provided to the ward pharmacist in the week prior to the intervention period. Participants admitted to an intervention ward during the study period will be screened by an in-hospital clinical staff member (ward nurses and/or pharmacists). Screening will involve checking whether the participant fulfils the inclusion/exclusion criteria. If the patient is eligible for inclusion, the clinical staff will ask potential participants or a substitute decision maker (where appropriate) if they are willing to have a researcher approach them to discuss participation. The staff will then notify the research team member who will proceed with the informed consent process and confirm eligibility.

Identification of participants for the retrospective group admitted to one of the target wards will be conducted via screening of electronic medical records of patients discharged from the target ward at least 1 week before the start of the intervention. That is, participants will be those eligible for inclusion consecutively discharged in reverse order, working back in time from 1 week prior to the start of the intervention until the desired sample size is achieved.

\section{Inclusion criteria}

- Age $\geq 70$ years old.

- DBI score $>0$ : taking $\geq 1$ regular medication with a sedative or anticholinergic effect prior to admission to the ward (ie, medications taken at home prior to admission or on transfer from another ward).

- Admitted to study ward $\leq 7$ days ago (from home or transfer).

- Written informed consent able to be obtained from participant or substitute decision maker.

- Able to communicate in English.
Exclusion criteria

- Expected discharge within 24 hours of recruitment or 48 hours of admission.

- Terminal phase of illness (expected to die during current admission) OR noted to be 'palliative care' (written in progress notes or consultation by the palliative care team).

- Usual residence outside Nova Scotia.

- Currently enrolled in another research study that involves administration of an experimental medication or a medication not approved by Health Canada.

\section{Intervention}

The intervention is multifaceted and involves a pharmacist-led medication optimisation initiative using an electronic decision support tool (the DBI Calculator). The DBI Calculator acts as a mechanism for pharmacists to identify and review potentially harmful anticholinergic and sedative medications. The intervention includes calculation of the DBI score and the DBI report that also acts as a communication and documentation tool both in hospital and on discharge. Provision of information to the participant or substitute decision maker on discharge is also standardised as part of the intervention. An overview of the study flow is shown in figure 1 and further outlined below.

Initially, the ward pharmacist will calculate a DBI score from the admission medication list or transfer medication reconciliation list, and a DBI report will be created. The admission medication list is referred to as a Best Possible Medication History (BPMH). A BPMH is a complete list of all medications (prescription and non-prescription, regular and when required) taken by the patient prior to admission to hospital. In the study hospitals, the BPMH is conducted by pharmacist or appropriately trained pharmacy resident, registered pharmacy technician, pharmacy practice assistant, physician, nurse or nurse practitioner. Regardless of who completed the BPMH, the intervention was delivered by the pharmacist. After creating the DBI report, the pharmacist will discuss their recommendations (using the report) with the healthcare team and then the participant/family as appropriate. All decisions about medication changes will be conducted as deemed appropriate by the healthcare team as would occur in regular care (and will not be dictated by the research team). On discharge, the DBI report and a letter about the study will be sent to the participant's family doctor (although no specific actions are required from them as a part of the study). If the participant has identified a regular pharmacy, a copy of the cover letter and DBI report will also be faxed to the pharmacy. A discharge medication list in the form of a medication calendar with details of changes made during admission will be provided to the participant/family prior to their discharge. A medication calendar is a list of the patients' medications and indication for each medication. The dose and frequency of each medication is noted in a morning, noon, supper and bedtime format resembling 
a calendar. The pharmacist will provide verbal information to the participant or substitute decision maker about the changes including information as to why the changes were made, whether there is any monitoring required (ie, follow-up with their doctor or symptoms to monitor) and if they should continue tapering of their medication following discharge.

The pharmacist will be instructed to incorporate use of the DBI Calculator into usual care for the intervention group. In usual care (ie, care received by the retrospective control group), patients have a BPMH conducted, medication reconciliation and review (by pharmacist, pharmacy resident or nurse) and sending of a discharge summary of treatment decisions to the participant's family doctor. In usual care, provision of verbal and/or written information to inpatients on discharge is variable and at the discretion of the pharmacist (can include verbal counselling, medication calendars and/or commercial handouts). While it is possible for healthcare professionals other than pharmacists to complete certain medicationrelated activities in hospital (such as nurses completing the BPMH) in both the intervention and control groups, this study will examine the addition of a pharmacist-led intervention to usual care (integrated into regular activities where possible).

\section{DBI Calculator}

The DBI Calculator is a web-based clinical decision support tool. The user (pharmacist) creates a new participant profile in the software and enters the participant's medication regimen/list (including doses and frequencies) as per the BPMH or transfer medication reconciliation. The medication list includes all prescription medications (regular and as needed (PRN: Pro Re Nata)) and can include over-the-counter medications. Medications with non-oral routes of administration (eg, patches and eye-drops) are also included. Within the DBI Calculator, there is a section to enter suspected sedative and anticholinergic adverse effects that the participant is experiencing. Additionally, for each medication entered, a free-text recommendation can be added, and an action (continue as clinically indicated, reduce dose, cease, no change, increase the dose, reduce dose with plan to cease or therapeutic substitution) can be assigned. Once this information is entered, a 'DBI Report' is created that includes all entered information plus the participant's DBI score with supporting information about the DBI. Ward pharmacists will be provided with education on using the DBI Calculator by a member of the research team prior to the start of the study.

The DBI for every participant is calculated using the equation,

$$
\mathrm{DBI}=\sum \frac{\mathrm{D}}{\mathrm{D}+\delta}
$$

where $\mathrm{D}$ is the daily dose and $\delta$ is the minimum adult licenced daily dose, as a surrogate for the dose required for $50 \%$ of the maximal effect. For this study, the minimum adult licenced daily dose was extracted from the product monograph in the Health Canada Drug Product Database if available and otherwise verified using a second Canadian drug information resource (Compendium of Pharmaceuticals and Specialties) ${ }^{2526} \mathrm{~A}$ score from 0 to 1 is calculated for each drug ingredient with anticholinergic and/or sedative effects based on the dose taken. ${ }^{81516}$ Medications with both anticholinergic and sedative effects were classified as anticholinergic. Complementary medications and medications that were prescribed 'when required' were excluded from the calculation. A difference in DBI of 0.5 is associated with clinically significant differences in physical function and falls. ${ }^{815}$

\section{Study sites}

The intervention is being investigated within hospital wards in Nova Scotia, Canada. The four wards, from three different hospitals, are purposely chosen to represent a variety of settings/contexts such as ward type and size of the hospital. The four sites are as follows: (1) geriatric ward in a large tertiary care centre in an urban setting, (2) surgical ward in a large tertiary care centre in an urban setting, (3) mixed general internal medicine and surgical ward in an urban small community hospital and (4) general internal medicine ward in a rural small community hospital.

\section{Outcomes}

See table 1 for details on how outcomes were assessed.

\section{Primary outcome}

- Proportion of inpatients in whom DBI score is decreased by $\geq 0.5$ at discharge, compared with on admission to hospital.

\section{Secondary outcomes}

- Proportion of inpatients in whom DBI score is decreased, unchanged or increased at 3 months after discharge, compared with at the time of hospital discharge.

- Total number of medications at hospital discharge and at 3 months after discharge.

- Proportion of inpatients who experience a clinical outcome during hospitalisation.

- New adverse drug reactions.

- Falls.

- Pressure ulcers.

- Proportion of inpatients who had a clinical outcome within 3 months of discharge

- One or more emergency department visits.

- Rehospitalisation (planned or unplanned).

- Mortality.

- Mean time (or median as appropriate) taken by clinical pharmacists to integrate the DBI Calculator into regular activities per participant.

- Exploration of the process of implementation and identification of factors that shaped implementation (outcome of mixed methods analysis). 
Table 1 Data collection and source of data

\begin{tabular}{|c|c|c|c|c|}
\hline Data & Source & Preintervention & Intervention & $\begin{array}{l}\text { 3-month } \\
\text { follow-up* }\end{array}$ \\
\hline $\begin{array}{l}\text { Sociodemographic data: age, sex, residence } \\
\text { (before and after discharge), education and } \\
\text { ethnicity }\end{array}$ & Progress notes. & $x$ & $x$ & \\
\hline $\begin{array}{l}\text { Medications at admission, discharge and } 3 \\
\text { months after discharge }\end{array}$ & $\begin{array}{l}\text { BPMH; DBI report; number of } \\
\text { medications from participant chart; } \\
\text { DIS† via HDNS. }\end{array}$ & $x$ & $x$ & $x$ \\
\hline
\end{tabular}

$\begin{array}{ll}\text { Frailty status prior to admission, } \text { CFS }^{12} & \text { Progress notes (if CFS score } \\ & \text { not provided in progress notes } \\ & \text { assessment will be made by research } \\ & \text { team using information in progress } \\ & \text { notes). }\end{array}$

Comorbidities at admission: number and ICD-10 codes; progress notes based $\times$ Charlson comorbidity index ${ }^{37}$

on medical history taken on admission with all active medical conditions counted.

\begin{tabular}{|c|c|c|c|c|}
\hline Reason for admission & Progress notes. & $x$ & $x$ & \\
\hline Falls in past year prior to admission & Participant/family. & & $x$ & \\
\hline Falls during admission & Progress notes. $\ddagger$ & $x$ & $x$ & \\
\hline $\begin{array}{l}\text { In-hospital new adverse drug reactions } \\
\text { (assessed using the Naranjo criteria }{ }^{38} \\
\text { independently by at least two research team } \\
\text { members) }\end{array}$ & Progress notes. $\neq$ & $x$ & $x$ & \\
\hline Length of hospitalisation & Progress notes. & $x$ & $x$ & \\
\hline In-hospital pressure ulcers & Progress notes. $\ddagger$ & $x$ & $x$ & \\
\hline $\begin{array}{l}\text { Patients attitudes towards deprescribing at } \\
\text { recruitment }\end{array}$ & $\begin{array}{l}\text { rPATD questionnaire }{ }^{39} \text { self-completed } \\
\text { at recruitment. }\end{array}$ & & $x$ & \\
\hline $\begin{array}{l}\text { Adverse drug withdrawal events during } \\
\text { hospitalization as defined by Graves et } a l^{40}\end{array}$ & Progress notes. $\neq$ & & $x$ & \\
\hline $\begin{array}{l}\text { Emergency department visits and readmission } \\
\text { to hospital }\end{array}$ & Electronic medical records. & & & $x$ \\
\hline Mortality & Vital statistics via HDNS. & & & $x$ \\
\hline
\end{tabular}

*3-month follow-up data for both intervention and control groups will be extracted when it becomes available from HDNS; the data are not available in real time.

†The Nova Scotia DIS is a province-wide system that contains a comprehensive medication profile for everyone who gets a prescription filled in a community pharmacy in Nova Scotia. Accuracy of the DIS at 3 months after discharge will be validated with a follow-up phone call to $10 \%$ of participants (randomly chosen) in the intervention group. A random number generator will be used in Excel to create a number for all participants. The data will then be sorted according to the random numbers and the top $10 \%$ of participants on the newly sorted list will be selected for each unit. The DIS is accessed via approval from HDNS.

$\ddagger$ Passive surveillance was used for these outcomes. Progress notes were reviewed in full after discharge to identify these outcomes including whether they were specifically noted as such by the care team (eg, adverse drug reactions).

BPMH, Best Possible Medication History; CFS, Clinical Frailty Scale; DBI, Drug Burden Index; DIS, Drug Information System; EQ-5D-5L, EuroQol-5 dimension-5 level; HDNS, Health Data Nova Scotia; ICD-10, International Classification of Diseases-Tenth Revision; MoCA, Montreal Cognitive Assessment; rPATD, Revised Patients' Attitudes Toward Deprescribing.

\section{Data collection}

Participants will be recruited, and baseline data will be collected during admission. Data collected and method of collection for the preintervention, intervention and 3 -month follow-up are outlined in table 1 . In addition to the data collected to assess outcomes (medications, adverse drug reactions, falls, pressure ulcers, emergency department visits, rehospitalisation and mortality), data will be collected to describe the participant sample and explore factors that might influence the process of implementation (eg, sociodemographic data, frailty status, comorbidities, reason for admission, length of hospitalisation, patient attitudes towards deprescribing, quality of life and cognition).

Basic details about the intervention implementation process will also be captured. Specifically, we will capture 
whether the DBI report was created and placed in the participant's chart, whether a copy of the DBI report was sent to the participant's family doctor and/or pharmacy and whether the pharmacist provided written and oral information on discharge. Where these elements are not conducted, a reason why will be sought where possible (ie, unexpected discharge).

\section{Analysis}

\section{Sample size and power}

The power calculation is based on preliminary results of an in-hospital, randomised controlled trial of the DBI Calculator conducted in Australia. ${ }^{27}$ To detect a significant difference in proportion of participants who had a reduction of their DBI score by $\geq 0.5$ points (a difference of 0.5 points is associated with clinically significant differences in physical function and falls), ${ }^{8} 1526$ participants are required per group per site (alpha $=0.05$, power $=0.8$ ). To detect a difference between adverse drug reactions found in the Australian study, a total of 157 participants are required in each group. Therefore, we aim to recruit 40 intervention participants per site: 160 intervention participants across all sites (and collect data for the same number of participants in the preintervention groups) to detect a change in prescribing at each site and a change in in hospital adverse drug reactions across all sites. To allow for dropouts, those transferred to different units during the study and missing follow-up data, we aim to recruit up to 50 participants per ward. Requests for the estimates used for sample size calculations, which are based on currently unpublished data, will be considered by the authors on a case-by-case basis.

\section{Quantitative data}

The difference in the primary outcome (proportion of participants with a reduction of $\geq 0.5$ in their DBI score between admission and discharge) between control and intervention groups will be analysed using a $\chi^{2}$ test. Secondary clinical outcomes (proportion of participants with new in-hospital adverse drug reactions, falls and pressure ulcers and one or more emergency department visits, rehospitalisation and mortality in the 3 months after discharge) will also be analysed using a $\chi^{2}$ test. Change in number of medications during admission between control and intervention groups will be analysed using appropriate parametric or non-parametric tests for continuous data depending on distribution of the data. Time taken by pharmacists to use the tool will be presented descriptively.

The relationship between DBI score following discharge and hospital readmission or mortality will be explored by unadjusted and adjusted (for age, sex and site) regression methods. In addition, other characteristics associated with the DBI score at baseline will be explored and considered in adjusted regression models.

Analyses will be conducted as intention to treat, with missing data imputed using multiple imputation methods. ${ }^{28}{ }^{29}$ Statistical significance is set at $\mathrm{p}<0.05$. Each participant will be assigned a number prior to data entry to maintain anonymity.

A subanalysis to determine if there is a differential effect of the intervention based on frailty and sex will also be conducted.

\section{Multiple case study}

To explore the process of implementation and identify elements that influenced implementation, a multiple case study (substudy) will be conducted. ${ }^{30}{ }^{31}$ The assumption of this study is that multiple factors (elements) will influence the success of implementation such as setting, clinician and participant factors. The data collected for this portion includes progress notes/information contained in the participant chart, medication information and interviews with the participant/family, ward pharmacist and other members of the medical team.

All participants in the intervention study will be informed that they may be invited to participate in a substudy that involves a follow-up telephone interview (within consent for main study). At each site, participants will be purposely sampled during the final 4 weeks of the intervention period: one who had a reduction in their DBI score and one who did not (or otherwise suitable participants to explore the success of implementation). Participants will also be asked at the time of consent whether they have a family member/friend who is involved with decisions about medications. If yes, the participant will be instructed to ask this person whether they are willing to be involved in the substudy, and if agreeing, will provide contact details of the individual to the researcher. The researcher will then phone the individual to gain informed consent.

Prior to beginning the intervention on the ward, the pharmacist and relevant staff members will be provided with information about the project, including the possibility of being asked to participate in this substudy. The pharmacist delivering the intervention and members of the medical team involved in the participant's care will be asked to provide informed consent for participation in this substudy where relevant for consented participants.

For the patient and family participants recruited for the multiple case study, it is anticipated that interviews will take approximately $30-60 \mathrm{~min}$ and will be conducted via phone at a time suitable to the participants. Interviews with medical team members will take approximately 15-30 min and conducted at a time suitable for them (in person or via phone with corresponding consent). For those who were involved with the care of more than one of the participants from the ward recruited for the multiple case study, they will only be interviewed once. Interviews will be audio recorded (then transcribed) and will be semistructured following an interview guide. NVivo will be used to manage data during collection and analysis. $^{30} 31$

Using a triangulation design model, the quantitative (intervention results) and qualitative (interviews) data will be collected simultaneously and analysed separately, 


\begin{tabular}{|c|c|c|}
\hline Intervention & Determinants & Outcomes \\
\hline $\begin{array}{l}\text { Electronic tool, DBI Calculator }(\text {, } \\
\text { integrated into pharmacist } \\
\text { medication optimisation } \\
\text { activities, plus standardised } \\
\text { communication between } \\
\text { patients and health care } \\
\text { professionals. } \\
\text { Target population } \\
\text { - } \geq 70 \text { years old } \\
\text { - DBI >0 (taking at least } 1 \\
\text { anticholinergic or sedative } \\
\text { drug) } \\
\text { - Males and females } \\
\text { - Frail and non-frail }\end{array}$ & $\begin{array}{l}\text { Treating healthcare team } \\
\text { For example: } \\
\text { - Composition of health care team - } \\
\text { pharmacist, medical doctors, nurses, } \\
\text { others } \\
\text { - Experience/specialty of healthcare } \\
\text { team members } \\
\text { Setting } \\
\text { For example: } \\
\text { - Size and location of the hospital } \\
\text { - Type of ward } \\
\text { - Cultural factors } \\
\text { Individual (patient characteristics) } \\
\text { For example: } \\
\text { - Age } \\
\text { - Sex or gender } \\
\text { - Frail and non-frail } \\
\text { - Attitudes towards deprescribing (rPATD) } \\
\text { Determinants to be identified and measured } \\
\text { through both quantitative analysis (e.g. male } \\
\text { versus female sex, frailty status) and through the } \\
\text { qualitative interviews }\end{array}$ & $\begin{array}{l}\text { Changes in medication use } \\
\text { BOTH a proxy marker for } \\
\text { improved patient care and } \\
\text { outcomes AND the success of } \\
\text { implementation } \\
\text { - DBI score } \\
\text { - Total number of medications } \\
\text { - Changes made to medications } \\
\text { (dose changes, discontinuations } \\
\text { and initiations) }\end{array}$ \\
\hline $\begin{array}{r}\text { Assumption: Inter } \\
\text { However - the effect of } \\
\text { Plus - the }\end{array}$ & $\begin{array}{l}\text { improvement in quality of medication use } \\
\text { ention may be influenced by the success of } \\
\text { utcomes may be directly influenced by indi }\end{array}$ & $\begin{array}{l}\text { ved clinical outcomes } \\
\text { ntation of the intervention } \\
\text { ated factors }\end{array}$ \\
\hline
\end{tabular}

Figure 2 Logic model. DBI, Drug Burden Index.

after which the two sets of results will be compared looking for consistency or contrast among the results. ${ }^{32}$ This analysis will address the third aim of this study and inform the secondary outcome of exploration of the process of implementation and identification of factors that shaped implementation. A logic model describing the potential relationship between the intervention, determinants and outcomes has been developed a priori and will guide this analysis (figure 2).

\section{Patient and public involvement}

A community representative has been engaged as a member of the research team and has provided input for several aspects of the study including confirmation of the research question relevance, participant recruitment and information, clinical outcomes and planning for dissemination of results. To ensure the community representative is engaged and supported in this role, a meeting with the project lead and the Patient EngagementCoordinator of the Maritime SPOR SUPPORT Unit and regular meetings with the project lead were held. At the conclusion of the project, a lay summary of the project and findings will be prepared for the general community audience, which will include community representative input and will be placed on the provincial health authority public website.

\section{Ethics and dissemination}

This study provides an electronic decision support tool and a process to enhance the activities of ward pharmacists that may lead to improved clinical outcomes for older adults. ${ }^{8151922}$ All changes being made to participants' medications (or any other aspects of their care) will be done by their medical team (with no control by the research team). The potential harms of medication discontinuation have been recently reviewed and include adverse drug withdrawal reactions and disruption of the doctor-patient relationship; however, the authors concluded that the potential for these harms was low when planned in conjunction with healthcare professional(s) and patient/family members and monitored. ${ }^{33}$ Any adverse effects of changes to medications will be monitored and documented by the medical team as part of the standard of care.

To reach researchers and healthcare professionals, traditional methods of dissemination will be used including peer-reviewed manuscripts and presentations at conferences and continuing education sessions.

\section{DISCUSSION}

Balancing the risks and benefits of medication use in older adults with multimorbidity is challenging. With more than 
half of older adults taking one or more unnecessary medications, reduction of polypharmacy and potentially inappropriate medications is imperative. Evidence on how best to execute and implement deprescribing strategies, including usefulness of clinical decision support tools to facilitate the process are emerging. However, the best method of deprescribing and the effect on clinical outcomes is still not clear. Deprescribing medications can be difficult due to barriers such as prescribing inertia, lack of process and feasibility. Despite this, $90 \%$ of patients report they are willing to have a medication deprescribed. ${ }^{34}$ Some of the most successful deprescribing initiatives described in the literature have incorporated clinical pharmacists and targeted specific medications. ${ }^{35} 36$

While we have designed a pragmatic study with the aim of integration into regular clinical activities to determine success of implementation and sustainability, this study has several limitations. Due to limitations of the DBI Calculator, the large number and variability of products available, and data sources, we are not able to include and collect information on all over-the-counter (non-prescription), herbal/natural health products and complementary medicines. Also, a 3-month follow-up may not be sufficient to determine long-term effects of the intervention. The DBI Calculator is currently a stand-alone program and as such integration with existing information technology systems and programme, such as prescribing software, is likely required for long-term sustainability. Due to the pragmatic nature of the study, participants, researchers and those conducting the intervention will not be blinded to the intervention. Additionally, due to the informed consent process required for the intervention participants, it is possible that older adults who are resistant to medication changes may self-select out of the study (even though willingness to have a medication deprescribed is not a requirement for inclusion). This type of self-selection will not occur in the control group as it is a retrospective sample (waiver of consent); this difference in consent processes between the intervention and control groups could therefore bias the results. As the control group data are collected retrospectively, only data that are routinely collected and documented during hospital admission can be included without possibility for clarification or checking with the participant. Finally, while this is an interventional study with a control group, there are possible factors that may confound the results. For example, change in season and change in medical and other staff between the control and the intervention periods.

\section{TRIAL STATUS}

This manuscript is based on trial protocol V.2.0 dated 9 November 2018. The pharmacist-led intervention to improve medication use in older inpatients using the DBI trial, opened to recruitment in February 2019 and is due to end in September 2019. Three-month outcome data will be collected retrospectively when mortality data become available through Health Data Nova Scotia (there is a lag in time between the relevant outcome period and when the data is available to be extracted). As of January 2020, we have recruited 45 intervention participants. Any protocol modifications will be communicated to relevant parties.

\section{Author affiliations}

${ }^{1}$ Geriatric Medicine Research, Nova Scotia Health Authority, Halifax, Nova Scotia, Canada

${ }^{2}$ Department of Pharmacy, Nova Scotia Health Authority, Halifax, Nova Scotia, Canada

${ }^{3}$ College of Pharmacy, Dalhousie University, Halifax, Nova Scotia, Canada

${ }^{4}$ Canadian Center for Vaccinology, Dalhousie University, Halifax, Nova Scotia, Canada

${ }^{5}$ Research Methods Unit, Nova Scotia Health Authority, Halifax, Nova Scotia, Canada ${ }^{6}$ Leslie Dan Faculty of Pharmacy, University of Toronto, Toronto, Ontario, Canada ${ }^{7}$ NHMRC Cognitive Decline Partnership Centre, Kolling Institute of Medical Research, Faculty of Medicine and Health, The University of Sydney, Sydney, New South Wales, Australia

${ }^{8}$ Clinical Pharmacology and Aged Care, Royal North Shore Hospital, Saint Leonards, New South Wales, Australia

${ }^{9}$ Department of Social and Preventive Medicine, Faculty of Medicine, Universite Laval, Québec city, Quebec, Canada

${ }^{10}$ Centre for Excellence on Aging of Quebec, Quebec Integrated University Centre for Health and Social Services of the National Capital, Québec city, Québec, Canada

${ }^{11}$ School of Health Administration, Dalhousie University, Halifax, Nova Scotia, Canada

${ }^{12}$ Department of Family Medicine, Nova Scotia Health Authority, Halifax, Nova Scotia, Canada

${ }^{13}$ Centre for Health Care of the Elderly, Nova Scotia Health Authority, Halifax, Nova Scotia, Canada

${ }^{14}$ Quality Use of Medicines Pharmacy Research Centre, School of Pharmacy and Medical Sciences, University of South Australia, Adelaide, South Australia, Australia

Twitter Lisa Kouladjian 0'Donnell @lisakouladjian and Emily Reeve @Reeve_ Research

Acknowledgements We would like to acknowledge the ongoing support provided by the clinical and research teams from Nova Scotia Health Authority (NSHA) Geriatric Medicine Research, Pharmacy Department and participating sites within NSHA Central Zone. We would also like to acknowledge Dr Colin Van Zoost for his input regarding the concept and design of the study and providing feedback on the presentation of the DBI Report. Lastly, we would like to thank our community representative, Marilyn Peers, for her provision of the patient, family and caregiver perspective, input regarding development of multiple case study interview guides and review of the protocol.

Contributors MED and ER contributed to the concept and design of this study and this manuscript, drafted the manuscript, revised the manuscript based on coauthor feedback and approved the final version for submission. SB, JI, KT, OK, LKO, HN, $\mathrm{SH}, \mathrm{CS}, \mathrm{MH}, \mathrm{AN}$ and KR contributed to the concept and design of this study and this manuscript, reviewed the manuscript and approved the final version for submission.

Funding This work was supported by the Canadian Frailty Network 2017 Catalyst Grant Competition, grant number CAT2017-13. In addition, funding for the follow-up phase was supported by the Nova Scotia Health Authority Research Fund and the Dalhousie (University) Pharmacy Endowment Fund. ER is supported by an NHMRC-ARC Dementia Research Development Fellowship.

Disclaimer The funders had no role in the study design; collection, management, analysis and interpretation of data; writing of the report; or the decision to submit the report for publication.

Competing interests The Drug Burden Index (DBI) Calculator Copyright 2019 The University of Sydney. All rights reserved. SH and LKO are the developers of the DBI Calculator.

Patient consent for publication Not required.

Ethics approval The intervention study protocol (including the multiple-case study) was approved by the Nova Scotia Health Authority (NSHA) Research Ethics Board (REB) on November 20, 2018 (File No. 1023962). The pre-intervention was approved July 19, 2018 (File No. 1023666). The first participant was consented on 6 February 2019. 
Provenance and peer review Not commissioned; externally peer reviewed.

Open access This is an open access article distributed in accordance with the Creative Commons Attribution Non Commercial (CC BY-NC 4.0) license, which permits others to distribute, remix, adapt, build upon this work non-commercially, and license their derivative works on different terms, provided the original work is properly cited, appropriate credit is given, any changes made indicated, and the use is non-commercial. See: http://creativecommons.org/licenses/by-nc/4.0/.

\section{ORCID iDs}

Marci Elizabeth Dearing http://orcid.org/0000-0002-6261-2411

Susan Bowles http://orcid.org/0000-0003-0821-3222

Jennifer Isenor http://orcid.org/0000-0003-1648-7362

Lisa Kouladjian 0'Donnell http://orcid.org/0000-0003-0927-7295

Sarah Hilmer http://orcid.org/0000-0002-5970-1501

Caroline Sirois http://orcid.org/0000-0003-3294-7883

Mohammad Hajizadeh http://orcid.org/0000-0002-4591-8531

Kenneth Rockwood http://orcid.org/0000-0002-6674-995X

Emily Reeve http://orcid.org/0000-0002-1405-999X

\section{REFERENCES}

1 Tannenbaum C. Effect of age, education and health status on community dwelling older men's health concerns. Aging Male 2012;15:103-8.

2 Tannenbaum C, Mayo N, Ducharme F. Older women's health priorities and perceptions of care delivery: results of the Wow health survey. CMAJ 2005;173:153-9.

3 Olsson IN, Runnamo R, Engfeldt P. Medication quality and quality of life in the elderly, a cohort study. Health Qual Life Outcomes 2011;9:95.

4 Muhlack DC, Hoppe LK, Weberpals J, et al. The association of potentially inappropriate medication at older age with cardiovascular events and overall mortality: a systematic review and meta-analysis of cohort studies. J Am Med Dir Assoc 2017;18:211-20.

5 Hajjar ER, Cafiero AC, Hanlon JT. Polypharmacy in elderly patients. Am J Geriatr Pharmacother 2007;5:345-51.

6 Scott IA, Hilmer SN, Reeve E, et al. Reducing inappropriate polypharmacy: the process of deprescribing. JAMA Intern Med 2015;175:827-34.

7 Nishtala PS, Salahudeen MS, Hilmer SN. Anticholinergics: theoretical and clinical overview. Expert Opin Drug Saf 2016;15:753-68.

8 Kouladjian L, Gnjidic D, Chen TF, et al. Drug burden index in older adults: theoretical and practical issues. Clin Interv Aging 2014;9:1503-15.

9 Reeve E, Wiese MD, Mangoni AA. Alterations in drug disposition in older adults. Expert Opin Drug Metab Toxicol 2015;11:491-508.

10 Ali-Melkkilä T, Kanto J, lisalo E. Pharmacokinetics and related pharmacodynamics of anticholinergic drugs. Acta Anaesthesiol Scand 1993;37:633-42.

11 Windle A, Elliot E, Duszynski K, et al. Benzodiazepine prescribing in elderly Australian general practice patients. Aust N Z J Public Health 2007;31:379-81.

12 Rockwood K, Song X, MacKnight C, et al. A global clinical measure of fitness and frailty in elderly people. CMAJ 2005;173:489-95.

13 Reeve E, Trenaman SC, Rockwood K, et al. Pharmacokinetic and pharmacodynamic alterations in older people with dementia. Expert Opin Drug Metab Toxicol 2017;13:651-68.

14 Gnjidic D, Hilmer SN, Blyth FM, et al. High-Risk prescribing and incidence of frailty among older community-dwelling men. Clin Pharmacol Ther 2012;91:521-8.

15 Hilmer SN, Mager DE, Simonsick EM, et al. A drug burden index to define the functional burden of medications in older people. Arch Intern Med 2007;167:781-7.

16 Hilmer SN. Calculating and using the drug burden index score in research and practice. Expert Rev Clin Pharmacol 2018;11:1053-5.

17 Jamieson HA, Nishtala PS, Scrase R, et al. Drug burden index and its association with hip fracture among older adults: a national population-based study. J Gerontol A Biol Sci Med Sci 2019;74:1127-33.

18 Anderson K, Stowasser D, Freeman C, et al. Prescriber barriers and enablers to minimising potentially inappropriate medications in adults: a systematic review and thematic synthesis. BMJ Open 2014;4:e006544.

19 Nishtala PS, Hilmer SN, McLachlan AJ, et al. Impact of residential medication management reviews on drug burden index in aged-care homes: a retrospective analysis. Drugs Aging 2009;26:677-86.
20 Reeve E, Gnjidic D, Long J, et al. A systematic review of the emerging definition of 'deprescribing' with network analysis: implications for future research and clinical practice. $\mathrm{Br} J \mathrm{Clin}$ Pharmacol 2015;80:1254-68.

21 Masnoon N, Shakib S, Kalisch-Ellett L, et al. Tools for assessment of the appropriateness of prescribing and association with patientrelated outcomes: a systematic review. Drugs Aging 2018;35:43-60.

22 Kouladjian L, Gnjidic D, Chen TF, et al. Development, validation and evaluation of an electronic pharmacological tool: the drug burden index Calculator(. Res Social Adm Pharm 2016;12:865-75.

23 Kouladjian O'Donnell L, Gnjidic D, Chen TF, et al. Integration of an electronic drug burden index risk assessment tool into home medicines reviews: deprescribing anticholinergic and sedative medications. Ther Adv Drug Saf 2019;10:204209861983247-71.

24 Wouters $\mathrm{H}$, van der Meer H, Taxis K. Quantification of anticholinergic and sedative drug load with the drug burden index: a review of outcomes and methodological quality of studies. Eur J Clin Pharmacol 2017;73:257-66.

25 Health Canada, government of Canada. Drug product database online query (2019), 2019. Available: https://health-products.canada. ca/dpd-bdpp/index-eng.jsp [Accessed 01 Aug 2019].

26 CPS [internet]. Ottawa (on): Canadian pharmacists association; c2016, 2019. Available: http://www.myrxtx.ca [Accessed 01 Aug 2019].

27 Australian new zealand clinical trials registry [internet]. Sydney (NSW): NHMRC clinical trials centre, University of Sydney (Australia). identifier. ACTRN12617000765325 minimising the functional burden of medications in older inpatients: implementation of the drug burden index, 2017. Available: https://anzctr.org.au/Trial/Registration/ TrialReview.aspx?id=372760\&isReview=true [Accessed 01 Aug 2019].

28 White IR, Daniel R, Royston P. Avoiding bias due to perfect prediction in multiple imputation of incomplete categorical variables. Comput Stat Data Anal 2010;54:2267-75.

29 White IR, Royston P, Wood AM. Multiple imputation using chained equations: issues and guidance for practice. Stat Med 2011;30:377-99.

30 Baxter P, Jack S. Qualitative case study methodology: study design and implementation for novice researchers. Qual. Rep. Nova Southeastern University; School of Social and Systematic Studies, 1990.

31 Yin R. Case study research: design and methods. Appl. Soc. Res. Methods 2003.

32 Creswell J. Research design: qualitative, quantitative, and mixed methods approaches. 2nd edn. Thousand Oaks: Sage, 2003.

33 Reeve E, Moriarty F, Nahas R, et al. A narrative review of the safety concerns of deprescribing in older adults and strategies to mitigate potential harms. Expert Opin Drug Saf 2018;17:39-49.

34 Reeve E, Wolff JL, Skehan M, et al. Assessment of attitudes toward deprescribing in older Medicare beneficiaries in the United States. JAMA Intern Med 2018;178:1673-80.

35 Sloane PD, Zimmerman S. Deprescribing in geriatric medicine: challenges and opportunities. J Am Med Dir Assoc 2018;19:919-22.

36 van der Meer HG, Wouters $\mathrm{H}$, Teichert M, et al. Feasibility, acceptability and potential effectiveness of an information technology-based, pharmacist-led intervention to prevent an increase in anticholinergic and sedative load among older community-dwelling individuals. Ther Adv Drug Saf 2019;10:204209861880588-81.

37 Sundararajan V, Henderson T, Perry C, et al. New ICD-10 version of the Charlson comorbidity index predicted in-hospital mortality. J Clin Epidemiol 2004;57:1288-94.

38 Naranjo CA, Busto U, Sellers EM, et al. A method for estimating the probability of adverse drug reactions. Clin Pharmacol Ther 1981;30:239-45.

39 Reeve E, Low L-F, Shakib S, et al. Development and validation of the revised patients' attitudes towards deprescribing (rPATD) questionnaire: versions for older adults and caregivers. Drugs Aging 2016;33:913-28.

40 Graves T, Hanlon JT, Schmader KE, et al. Adverse events after discontinuing medications in elderly outpatients. Arch Intern Med 1997;157:2205-10.

41 Herdman M, Gudex C, Lloyd A, et al. Development and preliminary testing of the new five-level version of EQ-5D (EQ-5D-5L). Qual Life Res 2011;20:1727-36.

42 Julayanont $\mathrm{P}$, Tangwongchai S, Hemrungrojn S, et al. The Montreal cognitive assessment-basic: a screening tool for mild cognitive impairment in illiterate and low-educated elderly adults. J Am Geriatr Soc 2015;63:2550-4. 